\title{
Interacting ultraviolet completions of four-dimensional gauge theories
}

\author{
Andrew D. Bond ${ }^{* \dagger}$ and Daniel F. Litim \\ Department of Physics and Astronomy, U Sussex, Brighton, BNI 9QH, U.K. \\ E-mail: a.bondesussex.ac.uk, d.litimesussex.ac.uk
}

\begin{abstract}
Ultraviolet fixed points provide an important means to define continuum limits of quantum field theories. In this contribution, we explain how interacting ultraviolet fixed points may arise in general four-dimensional gauge theories coupled weakly to scalar and fermionic matter fields. Our results derive from a systematic classification of all high- and low-energy fixed points in general gauge theories within perturbation theory. It is shown why and how Yukawa interactions provide a unique mechanism for the generation of perturbative ultraviolet fixed points. Phase diagrams for simple gauge theories are discussed to illustrate the main results. Some implications for particle physics beyond the Standard Model are indicated.
\end{abstract}

34th annual International Symposium on Lattice Field Theory

24-30 July 2016

University of Southampton, UK

${ }^{*}$ Speaker.

${ }^{\dagger}$ Supported by an STFC Studentship. 
Fixed points of the renormalisation group play an important role in particle physics. Ultraviolet (UV) fixed points are key for a fundamental definition of quantum field theories [1,2] such as in asymptotic freedom [3, 4] or in asymptotic safety [5]. Fixed points also serve to define the continuum limit in lattice versions of quantum field theory. Low energy fixed points appear prominently in phase transitions and the spontaneous breaking of symmetry. Fixed points may even play a role for physics beyond the Standard Model at the electroweak energy scale and above [6].

In this contribution, we discuss recent advances in the understanding of weakly interacting fixed points in $4 d$ gauge theories coupled to matter [7]. In general, finding interacting fixed points of quantum field theories is a challenging task. For a quantum field theory to possess a genuine fixed point, we must find values for its couplings such that all $\beta$-functions vanish simultaneously. The difficulty with this in practice is the fact that one has to deal with an infinite number of operators in the theory, each with a corresponding coupling. Furthermore, in interacting theories one does not know beforehand which operators, or combinations of operators, will correspond to relevant, marginal, or irrelevant directions $[8,9]$. One arena in which we may hope to be able to find reliable results is that of perturbation theory. If we restrict ourselves to such a domain, and search for fixed points which are weakly coupled, we gain two key advantages. Firstly, if interacting fixed points can be made sufficiently weakly coupled, the perturbative loop expansion will be trustworthy as we have information about the parametric size of higher loop corrections. Secondly, by ensuring that anomalous dimensions are small, the scaling dimensions of operators will remain close to their classical values, and the relevancy of operators will still be governed by their classical scaling dimension. This means that the only couplings whose relevancy we need to investigate are those with canonically dimensionless coupling constants, corresponding to classically marginal operators. Fortunately, the formal $\beta$-functions for such couplings are known for general four dimensional field theories $[10,11,12,13]$. Hence, by studying such general expressions, we can gain insights into the different types of perturbative fixed points that may arise in principle, and, moreover, identify candidate theories which realise them, in practice.

In this spirit, a complete classification of weakly coupled fixed points in general $4 d$ gauge theories including necessary and sufficient conditions for existence has recently been achieved in [7]. The main findings can be summarised as follows. Perturbative fixed points are either $(i)$ free (the Gaussian fixed point), or (ii) interacting in the gauge sector (Banks-Zaks fixed point) [14, 15], or (iii) simultaneously interacting in the gauge and the Yukawa sector (gauge-Yukawa fixed points), or (iv) products and combinations of $(i),(i i)$ and (iii). Scalar self interactions, if present, must take free or interacting fixed points of their own, compatible with vacuum stability, but are otherwise irrelevant to the fixed point in the gauge sector. It has also been proven that Banks-Zaks fixed points are invariably IR. In contrast, the Gaussian and the gauge-Yukawa fixed points can be either UV or IR, depending on the particulars of the field content. It is well-known that Gaussian UV fixed points play a central role for asymptotic freedom and the fundamental definition of quantum field theory $[3,4]$. In this light, weakly interacting gauge-Yukawa UV fixed points offer the intriguing prospect that fundamental and predictive quantum field theories may exist even beyond the paradigm of asymptotic freedom $[16,17]$.

To explain the unique dynamical mechanism underneath weakly interacting UV fixed points we investigate a simple gauge theory with gauge coupling $\alpha=g^{2} /(4 \pi)^{2}$ interacting with fermionic and/or scalar matter. Results straightforwardly generalise to more complex settings [7]. Within 
perturbation theory up to second order, the renormalisation group $\beta$-function $\beta \equiv \mu \frac{d \alpha}{d \mu}$ is given by

$$
\beta=-B \alpha^{2}+C \alpha^{3}+\mathscr{O}\left(\alpha^{4}\right)
$$

At one loop order the only possible fixed point is the free one, $\alpha^{*}=0$. Whether it is a UV or an IR fixed point depends on the sign of the one loop coefficient $B$. It is determined by the gauge and matter content of the theory and given by $[3,4,18]$

$$
B=\frac{2}{3}\left(11 C_{2}^{G}-2 S_{2}^{F}-\frac{1}{2} S_{2}^{S}\right)
$$

Here $S_{2}^{F, S}$ are the Dynkin indices of the fermion and scalar representations, and $C_{2}^{G}$ the quadratic Casimir of the adjoint representation. The contributions from the gauge bosons and the matter fields come with opposite signs, and so $B$ may in general take either sign. For a sufficiently small amount of matter, the gauge boson contributions dominate $(B>0)$ and the theory is asymptotically free. On the other hand, if there is enough matter, the contributions from the fermion and scalar fields dominate $(B<0)$ and the theory is IR free. In this case, higher loops are required to decide the fate of the theory in the UV. At two loop, (1) may display an interacting fixed point

$$
\alpha^{*}=\frac{B}{C}
$$

In order to have a physically viable theory we need $\alpha \geq 0$ so that $B \cdot C>0$, and in order for the fixed point to be perturbatively reliable, we must have $|B| \ll|C|$. Similarly to the one-loop case, the two-loop coefficient $C$ (given below) may take either sign, depending on whether gauge boson contributions dominate over matter field contributions $(C<0)$, or vice versa $(C>0)$. In the limiting cases of large or small amounts of matter fields, we have $B \cdot C<0$ and weakly interacting fixed points are absent. It has long been known that by partially offsetting the gauge boson contributions with matter, one may achieve $B, C>0[14,15]$ whereby the theory (1) displays the well-known Banks-Zaks IR fixed point (3). Much work has gone into perturbative and non-perturbative explorations of the related conformal window in view of physics beyond the SM [19, 20]. On the other hand, there are no known examples for which both coefficients take negative signs $B, C<0$, and in which case the fixed point would be UV. To explore this possibility, we write the two-loop term as

$$
C=\frac{2}{11}\left[2 S_{2}^{F}\left(11 C_{2}^{F}+7 C_{2}^{G}\right)+2 S_{2}^{S}\left(11 C_{2}^{S}-C_{2}^{G}\right)-17 B C_{2}^{G}\right] .
$$

The last term is non-negative if we arrange the matter content such that $B \leq 0$. The first term is manifestly non-negative, and we conclude that a theory with only fermions does not admit a weakly interacting UV fixed point [14]. The sign of the middle term becomes negative iff representations for scalar fields were to be found with a sufficiently small quadratic Casimir $C_{2}^{S}<\frac{1}{11} C_{2}^{G}$. Regrettably, however, this hope is futile: for any simple gauge theory, any non-trivial irreducible representation has a quadratic Casimir with $C_{2}^{R} \geq \frac{3}{8} C_{2}^{G}$ [7]. Moreover, this result imposes a strict bound on the coefficient $C$,

$$
C \geq C_{2}^{G}\left(\frac{28}{22} S_{2}^{F}+\frac{25}{22} S_{2}^{S}-\frac{34}{11} B\right)
$$

This result means that any IR free gauge theory $(B \leq 0)$ invariably has $C>0$. We conclude that a gauge-only interacting fixed point of the type (1), (3) can at best be IR, but never UV. 


\begin{tabular}{lcccc}
\hline case & gauge group & Yukawa & interacting FPs & type \\
\hline a) & simple & No & Banks-Zaks (BZ) & IR \\
\hline b) & semi-simple, no $U(1)$ factors & No & BZ and products thereof & IR \\
\hline \multirow{2}{*}{ c) } & simple & Yes & Banks-Zaks & IR \\
& simple & Yes & Banks-Zaks and gauge-Yukawa & IR \\
& simple or abelian & Yes & gauge-Yukawa (GY) & UV/IR \\
\hline d) & semi-simple w/ or w/o $U(1)$ & Yes & BZ, GY and products thereof & UV/IR \\
\hline
\end{tabular}

Table 1: Summary of weakly interacting fixed points in general gauge theories coupled to matter, detailing the availability of Banks-Zaks (BZ) or gauge-Yukawa (GY) type fixed points, or combinations and products thereof. The cases indicated under c) are illustrated in Fig. 1, see [7].

It is then natural to ask whether additional interactions may help generate an interacting UV fixed point? Owing to (5), when $B$ is small, the coefficient $C$ is of order unity. Any further contributions to the running of gauge couplings must appear at two-loop order, as otherwise couplings become non-perturbative. This rules out scalar quartic self-interactions, as these do not enter the running of the gauge couplings until at least three-loop. Making the gauge group semi-simple does not help either: the two-loop contributions of additional gauge couplings are strictly positive $[21,22]$. This leaves us with the only remaining option which are Yukawa couplings. They modify the running of gauge coupling (1) at two-loops, $\beta \rightarrow \beta+\Delta \beta$, with $\Delta \beta=-2 Y_{4} \alpha^{2}$ and

$$
Y_{4}=\operatorname{Tr}\left[\mathbf{C}_{2}^{\mathrm{F}} \mathbf{Y}^{\mathrm{A}}\left(\mathbf{Y}^{\mathrm{A}}\right)^{\dagger}\right] / \mathrm{d}(\mathrm{G}) .
$$

The set of Yukawa matrices $\mathbf{Y}^{\mathbf{A}}$ appears in the Lagrangian as $\sim \frac{1}{2}(4 \pi) \mathbf{Y}_{J K}^{A} \Phi^{A} \Psi_{J} \Psi_{K}$, and $d(G)$ being the gauge group dimension. As $Y_{4} \geq 0$, the contribution from Yukawa couplings to the gauge running is always negative, offering the prospect that these may counterbalance the gauge coupling contribution to negotiate for an interacting UV fixed point. To that end, we must examine the running of the Yukawa couplings and check whether they can develop a fixed point alongside the gauge coupling. To lowest non-trivial order, the $\beta$-function for the Yukawa matrix reads

$$
\beta_{J K}^{A}=E_{J K}^{A}(Y)-\alpha F_{J K}^{A}(Y),
$$

Notice that the gauge contribution arises with the opposite sign of the pure Yukawa contributions. Also, the quantity $E$ is cubic in the Yukawa matrices while $F$ is linear. Hence, any solution to the nullcline condition $\beta_{J K}^{A}=0$ must, by dimensional analysis, be of the form $\mathbf{Y}_{J K}^{A}=\frac{g}{4 \pi} \mathbf{C}_{J K}^{A}$ for some numerical matrices $\mathbf{C}^{\mathbf{A}}$ of order unity. Note that perturbativity in the gauge coupling entails perturbativity in the Yukawa coupling. If we project the gauge $\beta$-function onto this Yukawa nullcline, the net effect is that the two-loop term $C$ is replaced by an effective two-loop term $C^{\prime}$ given by

$$
C \rightarrow C^{\prime}=C-2 \operatorname{Tr}\left[\mathbf{C}_{2}^{\mathrm{F}} \mathbf{C}^{\mathrm{A}}\left(\mathbf{C}^{\mathrm{A}}\right)^{\dagger}\right] / \mathrm{d}(\mathrm{G})<\mathrm{C} .
$$

The projected gauge $\beta$-function then takes the form (1) subject to $C \rightarrow C^{\prime}$. The Yukawa-induced shift (8) has far-reaching implications. Firstly, if $B, C, C^{\prime}>0$ the theory is asymptotically free and 

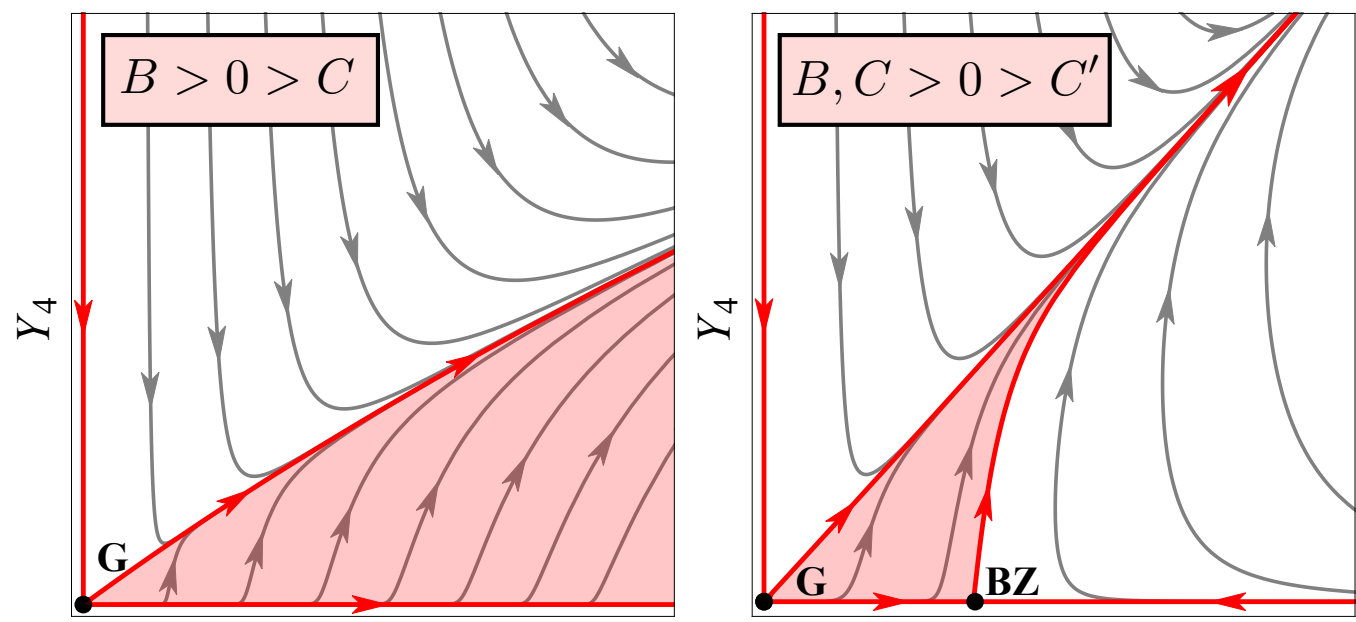

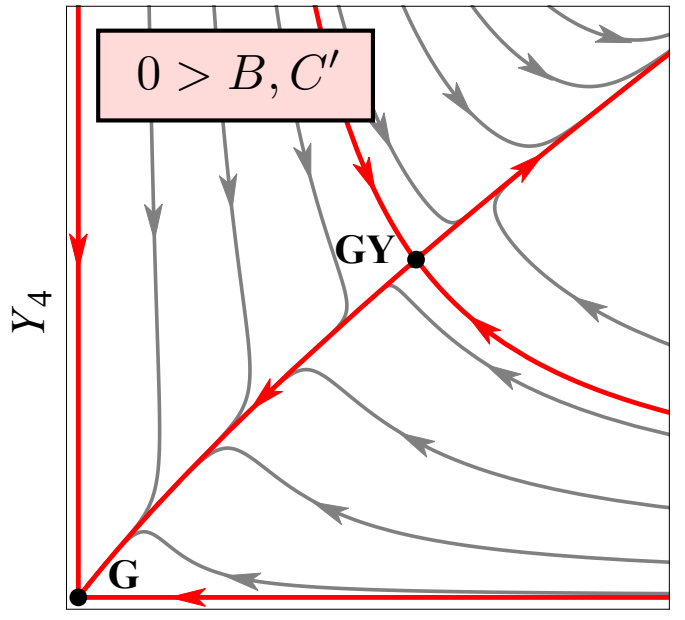

$\alpha$

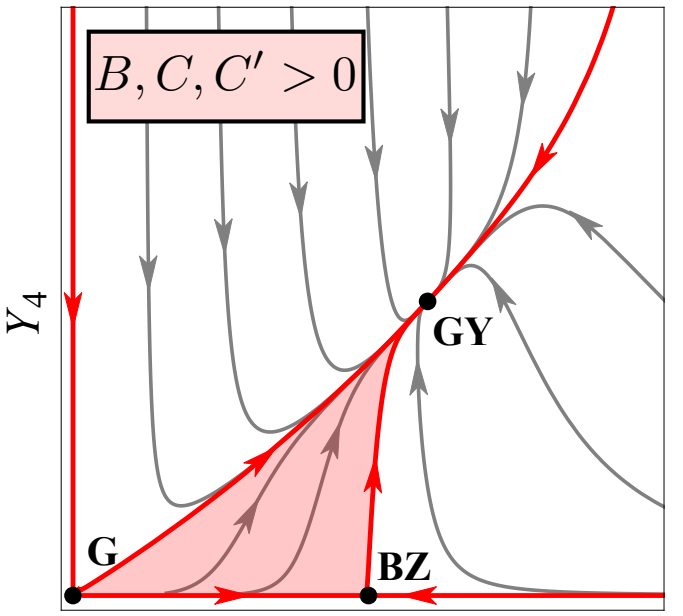

$\alpha$

Figure 1: Phase diagrams of simple gauge-Yukawa theories in dependence on the parameters $B, C$ and $C^{\prime}$. From top-left to bottom-left, clockwise: asymptotic freedom with Gaussian (G) UV fixed point $\left(B>0>C, C^{\prime}\right)$; asymptotic freedom with Banks-Zaks (BZ) fixed point $\left(B, C>0>C^{\prime}\right)$; asymptotic freedom with Banks-Zaks and gauge Yukawa (GY) fixed point $\left(B, C, C^{\prime}>0\right)$; asymptotic safety with gauge Yukawa fixed point $\left(C>0>B, C^{\prime}\right)$, see [7].

will display a novel interacting IR fixed point $\alpha^{*}=B / C^{\prime}$ besides the Banks-Zaks. Secondly, if $B<0$, we have $C>0$ and the fixed point (3) is not available. However, if Yukawa couplings can be found such that $C^{\prime}<0$, a novel UV fixed point arises at $\alpha^{*}=B / C^{\prime}$. No such fixed point can possibly exist without Yukawa interactions. This result establishes that Yukawa interactions, and hence elementary scalar fields and charged fermions, offer a unique mechanism for interacting UV fixed points to arise in weakly coupled gauge theories. Additional conditions arise from the fixed point in the scalar sector together with the stability of the vacuum $[16,17]$. The full range of weakly interacting fixed points for general four-dimensional field theories including necessary and sufficient conditions for existence are given in [7], A brief summary is given in Tab. 1. 
Next, we illustrate our findings for simple gauge theories coupled to matter on the level of their phase diagrams, see Fig.1, and in dependence on the parameters $B, C$ and $C^{\prime}$. Four basic cases arise. Asymptotically free gauge theories with $B>0>C$ have no weakly coupled fixed points, and their phase diagram displays the Gaussian UV fixed point together with UV free trajectories emanating out of it (red shaded areas). Theories with $B, C>0>C^{\prime}$ additionally develop a BanksZaks IR fixed point. Yukawa couplings are immaterial for this. Progressing towards theories with $B, C>C^{\prime}>0$ we now additionally observe a fully interacting IR gauge-Yukawa fixed point, besides the Banks-Zaks. The theory also comes out more strongly coupled at the gauge-Yukawa than at the Banks-Zaks fixed point. The main new effect in theories with $C^{\prime}>0$ as opposed to those with $C^{\prime}<0$ is that the UV-free trajectories are attracted into the gauge-Yukawa fixed point, which is genuinely attractive in both the gauge and the Yukawa directions. It characterises a second order phase transition between a symmetric phase and a phase with spontaneous symmetry breaking where the scalars acquire a non-vanishing vacuum expectation value. Finally, we consider simple or abelian gauge theories with $B, C^{\prime}<0$ where asymptotic freedom is absent. Most importantly, however, we observe a weakly interacting gauge-Yukawa fixed point which displays an attractive and a repulsive direction. The fixed point therefore qualifies as a UV completion for the theory. A concrete example of such a theory can be found in [16].

The discussion of phase diagrams generalises to more complex settings. Gauge theories with several independent Yukawa couplings will lead to several parameters $C^{\prime}$, which, depending on their magnitudes, may generate several gauge-Yukawa fixed points. Phase diagrams will then display an enhanced structure owing to additional cross-over phenomena amongst the various fixed points. An even richer pattern arises for theories with product gauge groups. For each gauge coupling individually our discussion based on the "diagonal" coefficients $B, C$ and $C^{\prime}$ applies. As a novel addition, theories may also display "off-diagonal" Banks-Zaks and gauge-Yukawa fixed points as well as fully interacting products thereof, depending on the details of the matter content. Moreover, each interacting fixed point naturally relates to a "conformal window" similar to those of QCD with fermionic matter [19]. Some of the fixed points of (product) gauge theories offer UV conformal windows around fixed points with exact asymptotic safety at weak coupling. It is therefore natural to speculate that some such models may qualify as UV completions for the Standard Model of particle physics.

In summary, we have identified all weakly interacting fixed points of four-dimensional gauge theories in the regime where gauge and matter fields remain good fundamental degrees of freedom. The virtue of our analysis are structural insights into the mechanisms for interacting fixed points together with strict perturbative control. We thereby have established that weakly interacting low-energy fixed points are either of the Banks-Zaks or gauge-Yukawa type, or combinations and products thereof (Tab. 1). They offer a rich spectrum of phenomena including phase transitions and the spontaneous breaking of (chiral) symmetry. We have also seen that weakly interacting high-energy fixed points are invariably of the gauge-Yukawa type. Hence, interacting UV fixed points necessarily require elementary scalar fields such as the Higgs. It is quite intriguing that the dynamical mechanism to tame the notorious Landau poles of general infrared free gauge theories is unique, and, owing to group-theoretical limitations (5), exclusively delivered through Yukawa interactions. We conclude that our findings open a window of opportunities towards perturbative UV completions of the Standard Model beyond the paradigm of asymptotic freedom. 


\section{References}

[1] K. G. Wilson, Renormalization group and critical phenomena. 1. Renormalization group and the Kadanoff scaling picture, Phys.Rev. B4 (1971) 3174-3183.

[2] K. G. Wilson, Renormalization group and critical phenomena. 2. Phase space cell analysis of critical behavior, Phys.Rev. B4 (1971) 3184-3205.

[3] D. J. Gross and F. Wilczek, Ultraviolet Behavior of Nonabelian Gauge Theories, Phys. Rev. Lett. 30 (1973) 1343-1346.

[4] H. D. Politzer, Reliable Perturbative Results for Strong Interactions?, Phys. Rev. Lett. 30 (1973) 1346-1349.

[5] S. Weinberg, Ultraviolet divergences in quantum theories of gravitation, . In General Relativity: An Einstein centenary survey, ed. S. W. Hawking and W. Israel, 790- 831.

[6] D. F. Litim, Renormalisation group and the Planck scale, Phil. Trans. Roy. Soc. Lond. A369 (2011) 2759-2778, [1102.4624].

[7] A. D. Bond and D. F. Litim, Theorems for Asymptotic Safety of Gauge Theories, 1608.00519.

[8] K. Falls, D. F. Litim, K. Nikolakopoulos, and C. Rahmede, A bootstrap towards asymptotic safety, 1301.4191.

[9] K. Falls, D. F. Litim, K. Nikolakopoulos, and C. Rahmede, Further evidence for asymptotic safety of quantum gravity, Phys. Rev. D93 (2016), no. 10 104022, [1410.4815].

[10] M. E. Machacek and M. T. Vaughn, Two Loop Renormalization Group Equations in a General Quantum Field Theory. 1. Wave Function Renormalization, Nucl.Phys. B222 (1983) 83.

[11] M. E. Machacek and M. T. Vaughn, Two Loop Renormalization Group Equations in a General Quantum Field Theory. 2. Yukawa Couplings, Nucl. Phys. B236 (1984) 221-232.

[12] M. E. Machacek and M. T. Vaughn, Two Loop Renormalization Group Equations in a General Quantum Field Theory. 3. Scalar Quartic Couplings, Nucl. Phys. B249 (1985) 70-92.

[13] M.-x. Luo, H.-w. Wang, and Y. Xiao, Two loop renormalization group equations in general gauge field theories, Phys. Rev. D67 (2003) 065019, [hep-ph/ 0211440 ].

[14] W. E. Caswell, Asymptotic Behavior of Nonabelian Gauge Theories to Two Loop Order, Phys. Rev. Lett. 33 (1974) 244.

[15] T. Banks and A. Zaks, On the Phase Structure of Vector-Like Gauge Theories with Massless Fermions, Nucl. Phys. B196 (1982) 189.

[16] D. F. Litim and F. Sannino, Asymptotic safety guaranteed, JHEP 12 (2014) 178, [1406.2337].

[17] D. F. Litim, M. Mojaza, and F. Sannino, Vacuum stability of asymptotically safe gauge-Yukawa theories, JHEP 01 (2016) 081, [1501.03061].

[18] D. J. Gross and F. Wilczek, Asymptotically Free Gauge Theories. 1, Phys. Rev. D8 (1973) 3633-3652.

[19] L. Del Debbio, The conformal window on the lattice, PoS Lattice2010 (2014) 004, [1102 . 4066 ].

[20] D. D. Dietrich and F. Sannino, Conformal window of $S U(N)$ gauge theories with fermions in higher dimensional representations, Phys. Rev. D75 (2007) 085018, [hep-ph / 0611341$].$

[21] D. R. T. Jones, Two Loop beta Function for a G(1) x G(2) Gauge Theory, Phys. Rev. D25 (1982) 581.

[22] F. del Aguila, G. D. Coughlan, and M. Quiros, Gauge Coupling Renormalization With Several U(1) Factors, Nucl. Phys. B307 (1988) 633. [Erratum: Nucl. Phys.B312,751(1989)]. 